\title{
Efectos directos e indirectos entre estilos de pensamiento, estrategias metacognitivas y creatividad en estudiantes universitarios
}

\author{
Calixto Gutierrez-Braojos*, Purificación Salmeron-Vilchez, Ana Martin-Romera y Honorio Salmerón
}

Departamento de Métodos de Investigación y Diagnóstico en Educación. Universidad de Granada, España

\begin{abstract}
Resumen: Desde la psicología educativa se han generado estudios que relacionan los estilos de pensamiento con las estrategias metacognitivas y la creatividad. Aunque existe un cuerpo empírico que explicita relaciones de dependencia y/o predictivas entre estos constructos, no hemos hallado estudios que analicen los efectos directos e indirectos que se establecen entre ellos. Así, el objetivo en este estudio fue probar un modelo teórico mediante modelización con ecuaciones estructurales para estudiar dichos efectos. Participaron 197 estudiantes universitarios. Los instrumentos usados fueron: i) el Inventario de Estilos de Pensamiento (TSI-R) (Sternberg, Wagner y Zhang, 2003) para medir los estilos de pensamiento; ii) La Escala de Estrategias de Aprendizaje (ACRA) (Román y Gallego, 2001) para medir las estrategias metacognitivas; iii) El Test de Inteligencia Creativa (CREA) (Corbalán Berná et al., 2003) para medir la creatividad. Los resultados obtenidos indican que: i) Los estilos de pensamiento judicial y legislativo (Sternberg, 1998) contribuyen de manera positiva y directamente a uso de estrategias metacognitivas e indirecta y positivamente a la creativi dad; ii) las estrategias metacognitivas contribuyen de manera directa y positivamente a la creatividad. Sin embargo, no se ha encontrado una relación directa entre los estilos de pensamiento y la creatividad.

Palabras clave: Estilos de pensamiento; estrategias metacognitivas; creatividad; Educación Superior.
\end{abstract}

Title: Direct and indirect effects between thinking styles, metacognitive strategies and creativity in college students.

Abstract: Previous studies from the field of Educational Psychology have indicated that thinking styles are related to metacognitive strategies and creativity. Although, there is a body of empirical studies which explains the relationship and/ or predictive relations between these constructs, we have not found studies examining the direct and indirect effects that arise between them. Thus, the objective of this study was to test a theoretical model using structural equation modeling to study these effects. Thus, the objective of this study was to test a theoretical model using structural equation modeling to study these effects. Participants in the present study were 197 university students. Instruments used were: i) the Thinking Styles Inventory (TSI-R) (Sternberg, Wagner \& Zhan, 2003) to measure thinking styles; ii) The Learning Strategies Scale (ACRA) (Roman \& Gallego, 2001) to measure the metacognitive strategies, iii) The Test of Creative Intelligence (CREA) (Corbalan Berna et al., 2003) to measure creativity. The results indicate that: i) The judicial and legislative thinking styles (Sternberg, 1998) contribute to the use of metacognitive strategies directly and in a positive way, and these styles contribute to the creativity indirectly and in a positive way; ii) metacognitive strategies contribute to the creativity directly and in a positively way. However, not found a direct relationship between thinking styles and creativity.

Keywords: Thinking style; metacognitive strategies; creativity; Higher Education.

\section{Introducción}

La Teoría del Autogobierno Mental (Sternberg, 1988; Zhang y Sternberg, 2005) es una de las más recientes sobre los estilos intelectuales (Zhang, 2010a). Los estilos de pensamiento, o aspectos estilísticos de la cognición, son un elemento clave de la teoría del autogobierno mental y explicitan las diferentes formas que tienen las personas de utilizar sus habilidades intelectuales (Gringorenko y Sternberg, 1997; Sternberg, 1998). La teoría describe trece estilos de pensamiento agrupados en cinco dimensiones: i) funciones (estilos legislativo / judicial / ejecutivo); ii) formas (estilo monárquico / jerárquico / oligárquico / anárquico); iii) niveles (estilo global / local); iv) alcance (estilo interno / externo); v) inclinaciones (estilo liberal / conservador).

Esta investigación se centra en el estudio de las funciones de estilos de pensamiento, entendidas como preferencias que tienen las personas a la hora de canalizar y organizar el pensamiento. Como apunta Sternberg (1998) estas funciones "no se refieren a una aptitud sino a cómo utilizamos las aptitudes que tenemos" (p. 38). Esta teoría establece tres tipos de funciones de los estilos de pensamiento: legislativo, ejecutivo y judicial. Las personas con cierta tendencia hacia la

* Dirección para correspondencia [Correspondence address]: Calixto Gutierrez Braojos, Departamento de Métodos de Investigación y Diagnóstico en Educación. Facultad de Educación y Humanidades, Universidad de Granada. C/ El Greco S/N 51002 (España). E-Mail: calixtogb@ugr.es función legislativa se caracterizan por una preferencia hacia tareas que permitan establecer sus propias directrices sobre cómo realizarlas. Las personas con cierta tendencia hacia la función ejecutiva se caracterizan por una predilección por tareas que contengan instrucciones y directrices claras sobre lo que deben hacer para realizarlas. Y las personas con tendencia hacia la función judicial se caracterizan por cierta inclinación por tareas que requieran actividad mental crítica.

Existen investigaciones que han analizado la relación de este constructo con otros tópicos vinculados a los procesos de aprendizaje, como por ejemplo, las estrategias metacognitivas (e.g. Zhang, 2010a) y la creatividad (e.g. López Martínez y Martín Brufau, 2010) en contextos universitarios. Además, otros estudios han analizado la relación entre las estrategias metacognitivas y la creatividad (Runco y Okuda, 1988). Sin embargo, solamente hemos encontrado un artículo empírico que analizan conjuntamente la relación entre dichos constructos (Zhang, 2010a). Esta investigación utiliza un procedimiento de análisis por regresión y solo ofrece relaciones únicas entre variables dependientes e independientes. Por ello, son necesarios otros estudios que ofrezcan un análisis complejo de cómo estos constructos se relacionan entre sí, especificando los efectos directos e indirectos que se generan entre ellos.

En este artículo se propone y analiza un modelo teórico que relaciona los estilos de pensamiento, las estrategias metacognitivas y la creatividad mediante modelización con ecuaciones estructurales (Figura 1). En base a evidencias previas, 
se postula que los estilos de pensamiento contribuyen directa y positivamente al uso de estrategias metacognitivas y a la creatividad. También que las estrategias metacognitivas contribuyen directa y positivamente a la creatividad.

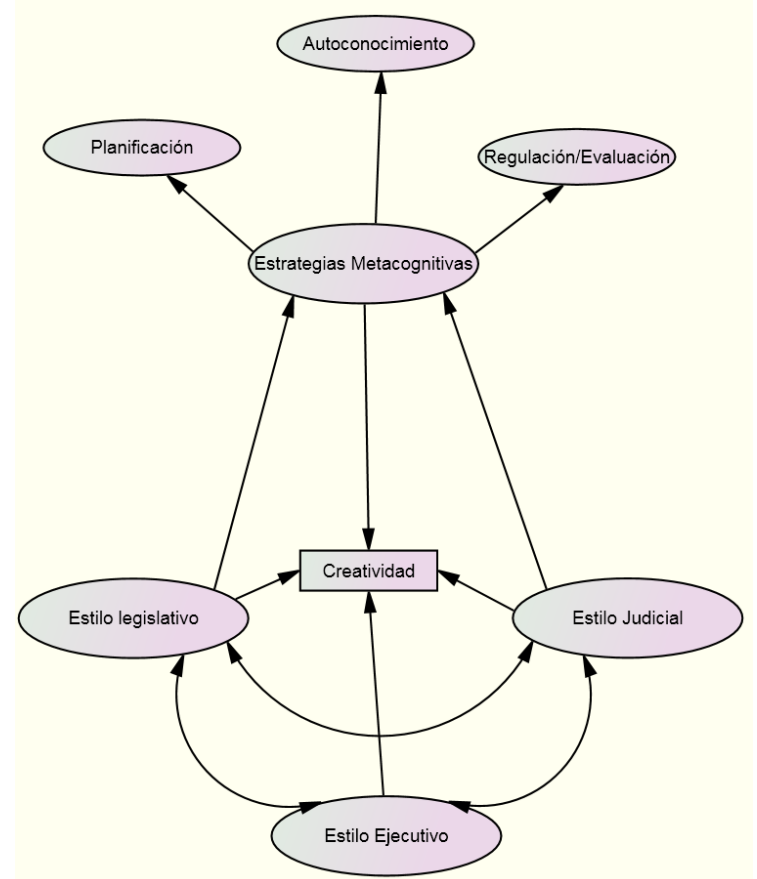

Figura 1. Modelo de estructura: estilos de pensamiento, estrategias metacognitivas y creatividad

\section{Estilos de pensamiento y creatividad}

La creatividad es fundamental para generar transformación, evolución, innovación y desarrollo social. Como señalo Einstein (1985) "sin personalidades creadoras que piensen por sí mismas es tan impensable el desarrollo de la comunidad como lo sería el desarrollo del individuo fuera del ámbito comunitario" (pp. 27-28). En este sentido, una de las responsabilidades inherentes de las instituciones universitarias es proporcionar, desde la excelencia docente e investigadora, la formación de aprendices que posteriormente serán profesionales transformadores, reflexivos y críticos. La creatividad se refiere a la generación de nuevas ideas y la traducción de estas ideas en nuevos productos útiles (Guilford, 1967; Sternberg y Lubart, 1996), y la persona creativa es aquella cuyos pensamientos o acciones generan avances en un dominio de conocimiento, o establecen uno nuevo (Csikszentmihalyi, 1997).

Muchos trabajos hipotetizan que múltiples componentes deben converger para que la creatividad se produzca (e.g. Amabile, 1983; Sternberg, 1985). Por ello, la creatividad es un constructo polémico y complejo, difícil de medir y de evaluar (Sternberg, 1999). En este estudio, para su medida, nos centramos en la perspectiva psicométrica en la que son pioneros los trabajos de Guilford (1950) y Torrance (1966).
Desde esta perspectiva se aplican test normativos para la evaluación del pensamiento divergente (Oliveira et al., 2009) como por ejemplo, el test de inteligencia creativa (CREA) (Corbalán Berná et al., 2003).

El pensamiento divergente es un componente necesario en la creatividad (Clapham y King, 2010) y suele utilizarse para estimar el potencial creativo (Runco, 1993, 2006). Este se refiere a la capacidad de las personas para generar gran cantidad de ideas alternativas que además han de ser originales y diferentes entre sí ante una demanda determinada (Runco, 2006; Zhang, 2010b). Según Sternberg y Lubart (1997) los estilos de pensamiento pueden tanto fomentar como dificultar el pensamiento creativo.

Según diferentes estudios, dos son los estilos que se vinculan al pensamiento divergente, el legislativo y el judicial (Sternberg, 1999; Zhang y Sternberg, 2005, Zhang, 2004, 2010b). Sin embargo, también existen estudios que relacionan positivamente el estilo de pensamiento ejecutivo con la creatividad (Zhang, 2010a) y estudios que indica que esta relación es significativa y negativa (Zhang, 2004).

En estudios con la población española, se han encontrado resultados similares. Así, López Martínez y Martín Brufau (2010) concluyen que los estudiantes con una alta puntuación en pensamiento divergente obtienen unas puntuaciones más altas en estilo legislativo. Los estudiantes con una puntuación moderada en el pensamiento divergente obtienen puntuaciones altas en el estilo judicial. Y los estudiantes que puntuaron bajo en el test de pensamiento divergente presentaban un perfil de pensamiento ejecutivo. La media de puntuaciones en el estilo de pensamiento judicial fue ligeramente más elevada en los estudiantes con un nivel de creatividad alto que en los estudiantes que manifiestan un nivel de creatividad medio o bajo. De esta forma, estos autores encuentran un perfil de los estudiantes creativos (pensamiento divergente) que se caracteriza por puntuaciones altas en el estilo de pensamiento legislativo, puntuaciones bajas en el estilo de pensamiento ejecutivo y puntuación moderada en estilo de pensamiento judicial.

En la misma línea, Almansa Martínez y López Martínez (2010), en su estudio sobre estilos de pensamiento y pensamiento divergente en estudiantes de enfermería, hallaron que aquellos estudiantes con unas puntaciones bajas en pensamiento divergente presentaban un estilo ejecutivo. Sin embargo, no encontraron relaciones significativas entre el resto de estilos de pensamiento (legislativo y judicial) y el pensamiento divergente.

En resumen, la función de los estilos de pensamiento que más se relaciona con la creatividad es la legislativa.

\section{Los Estilos de pensamiento y las estrategias metacognitivas}

Desde la psicología educativa se postula un modelo jerárquico de los procesos cognitivos (Denckla, 2007). En la cúspide de dicha jerarquía se encuentran la función ejecutiva, que en los procesos de aprendizaje suelen denominarse metacogni- 
ción (Flavell, 1977). Así, las estrategias metacognitivas son definidas como la autocomprobación consciente y sistemática de la acción respecto a la meta, así como la selección y aplicación de estrategias, cuando las circunstancias lo requieran (O’Neil y Abedi, 1996). Para Román y Gallego (2001) estas estrategias metacognitivas "suponen y apoyan, por una parte, el conocimiento que una persona tiene de los propios procesos, en general, y de estrategias cognitivas de aprendizaje en particular y, por otra, la capacidad de manejo de las mismas" (p.15). Según estos autores, las estrategias de autoconocimiento se conforman de: i) conocimiento declarativo (saber qué hacer); ii) conocimiento procedimental (saber cómo hacer); iii) conocimiento condicional (saber cuándo y por qué hacer). Lo importante para el estudiante, es pues i) saber detectar y seleccionar qué estrategias son útiles en cada circunstancia; ii) saber cómo y cuándo se deben aplicar esas estrategias; y iii) saber comprobar eficientemente la eficacia de la estrategia empleada. Las estrategias de automanejo requieren de: i) una planificación o establecimiento de metas en un contexto de aprendizaje dado; ii) una evaluación respecto al grado de consecución alcanzado respecto a los objetivos planificados; iii) regulación y rectificación en función de si se alcanzan, o no, dichos objetivos..

Respecto a la relación entre las estrategias metacognitivas con los estilos de pensamiento, se han encontrado solo dos estudios dirigidos a la población de estudiantes. El primero (Zhang, 2010a), en una muestra de estudiantes universitarios (entre 17 y 32 años) concluye que solo los estudiantes con estilo ejecutivo usan estrategias metacognitivas (consciencia del conocimiento y regulación de la cognición). Los estudiantes con un estilo legislativo emplean exclusivamente estrategias relacionadas con la consciencia del conocimiento. Y los estudiantes con un estilo judicial no usan estrategias metacognitivas (consciencia del conocimiento y regulación de la cognición). Por otro lado, el estudio de Caycho (2009) en una muestra de estudiantes (con una edad comprendida entre 15 y 19 años), concluye que el uso de estrategias metacognitivas se relaciona con las tres funciones de estilos de pensamiento.

Además, otros estudios, basados en la línea de investigación de los enfoques de aprendizaje, han relacionado indirectamente las estrategias metacognitivas con los estilos de pensamiento. Desde esta línea de investigación se establecen básicamente dos enfoques de aprendizaje: el superficial y el profundo (Entwistle y Ramsden, 1983). El enfoque profundo se relaciona con: el uso de estrategias profundas para el aprendizaje constructivista (Biggs, 1987; Hernández Pina y Hervás, 2005), la aplicación de estrategias metacognitivas (Vermetten, Vermunt y Lodewijks, 1999; MartínezFernández, Villegas y Martínez Torres, 2004) y una tendencia hacia las funciones legislativas y judicial de los estilos de pensamiento, pero no hacia la función ejecutiva (Zhang, 2000; Zhang y Sternberg, 2000; Rosário et al., 2005).

En suma, hay un insuficiente cuerpo de investigaciones sobre la relación entre estos constructos y las observadas ofrecen conclusiones inconsistentes $y / o$ controvertidas que podrían explicarse en función del enfoque teórico, los instrumentos de medida de la edad de los participantes, variables culturales, contextuales...

\section{Estrategias metacognitivas y creatividad}

Desde el enfoque metacognitivo (Pesut, 1990), el pensamiento creativo es considerado como producto de un proceso autorregulador metacognitivo. Según Runco (2007), el pensamiento creativo se facilita cuando se activan intencionalmente tácticas (conocimientos procedimentales) supeditadas por habilidades metacognitivas con el propósito de alcanzar un producto creativo o resolución creativa a un problema en un contexto determinado. Dicho de otro modo, la metacognición y la autorregulación es el conocimiento condicional que gobierna estas tácticas o conocimiento procedimental. Comprender el pensamiento creativo exclusivamente desde el prisma procedimental, implicaría obviar el carácter intencional de la capacidad creativa. Así, este enfoque defiende que son las experiencias metacognitivas las que permitirán entender y regular el comportamiento para alcanzar una meta creativa (Mednick, 1962; Bransford y Stein, 1984; Armbruster, 1989; Pesut, 1990; Rothenberg, 1990; Nickerson, 1999; Runco, 2004).

Según las conclusiones en varios estudios, estas habilidades metacognitivas favorecen el pensamiento creativo por varias razones:

i) Favorecen la activación del conocimiento previo (Bernal, 2001; Mednick, 1962).

ii) Facilitan la compenetración con el estado de las representaciones cognitivas del conocimiento, (Armbruster, 1989). En otras palabras estos pueden saber si su conocimiento es completo, rico y suficientemente flexible para proporcionar el potencial de reestructuración creativa.

iii) Son útiles en la manipulación consciente de varias ideas simultáneas. Dicho de otro modo, la metacognición es un requisito del pensamiento homoespacial que resulta clave en el pensamiento creativo (Rothenberg, 1990).

iv) Pueden ser particularmente útiles para el control o la regulación de la codificación de representaciones flexibles del conocimiento (Armbruster, 1989). Así, ellos pueden conocer y usar estrategias metacognitivas que garanticen un conocimiento flexible.

v) Son necesarias en la fase de verificación del proceso creativo. Esto es, la metacognición juega un papel relevante en cuanto a la evaluación del producto (Armbruster, 1989).

Sin embargo, las investigaciones dirigidas a estudiar la relación entre ambos constructos presentan conclusiones controvertidas en función de la disciplina de estudio. Los estudios específicos en la disciplina de Bellas Artes concluyen que las altas habilidades metacognitivas se asocian negativamente con diferentes medidas de la creatividad (Furnham, Zhang, y Chamorro-Premuzic, 2006; Wolfradt y Pretz, 
2001). Los estudios realizados en disciplinas diferentes a Bellas Artes y en diferentes dominios específicos, (e.g. resolución de problemas, elaboración de proyectos) concluyen que altas habilidades metacognitivas favorecen el proceso y producto creativo (Chand y Runco, 1992; Feldhusen y Goh, 1995; Harrington, 1975; Jausovec, 1994; Rucinski y Arredondo, 1994; Runco y Okuda, 1988; Zampetakis, Bouranta y Moustakis, 2010).

Aceptando el cuerpo de investigaciones documentadas, y posicionándonos dentro del enfoque metacognitivo, consideramos posible generar productos creativos independientemente de las capacidades metacognitivas. Una persona podría generar productos creativos de manera casual en un contexto histórico-cultural determinado. Esto no implica que consideremos a las habilidades metacognitivas como el único factor relevante en pensamiento creativo. Como señala Barron (2007), la creatividad no requiere exclusivamente de habilidades metacognitivas sino que es "un reflejo de cognición, metacognición, actitud, motivación, afecto, disposición y temperamento" (p. 320).

En suma, consideramos que existen evidencias empíricas que relacionan dichos constructos, aunque no explicitan los efectos directos e indirectos que se generan entre ellos. Por ello, el presente estudio se dirige a generar un cuerpo de conocimiento que permita profundizar sobre el tipo de relaciones entre dichos constructos. Para ello, se analiza un modelo teórico que explicite dichos efectos. Este modelo comprende los estilos de pensamiento (funciones de los estilos de pensamiento) como una variable latente exógena, las estrategias metacognitivas como una variable latente endógena, y en último lugar, la creatividad se comprende como la variable dependiente explicada por las variables latentes endógenas y exógenas. Específicamente con este modelo teórico, fundamentado en el cuerpo de investigaciones, se apuntan las siguientes hipótesis:

i) Relaciones entre las funciones de los estilos de pensamiento y las estrategias metacognitivas: Los estilos de pensamiento legislativo y judicial contribuyen positivamente al uso de estrategias metacognitivas; y un estilo de pensamiento ejecutivo no contribuye al uso de estrategias metacognitivas (Zhang, 2000; Zhang y Sternberg, 2000).

ii) Relaciones entre las funciones de los estilos de pensamiento y la creatividad: Los estilos de pensamiento legislativo y judicial contribuye positivamente y directa e indirectamente a la capacidad creativa, y un estilo ejecutivo se corresponde con una baja capacidad creativa (López Martínez y Martín y Brufau, 2010; Almansa Martínez y López Martínez, 2010; Zhang, 2010a).

iii) Relaciones entre las estrategias metacognitivas y a la capacidad creativa: El uso de estrategias metacognitivas contribuye directamente y positivamente a la capacidad creativa (Chrodak y Prieto 2010; Barak, 2010; Zhang, 2010a)

\section{Método}

\section{Participantes}

Los participantes fueron 197 estudiantes universitarios españoles ( $81.2 \%$ mujeres y $18.8 \%$ hombres) matriculados en el cuarto año de la carrera de Psicopedagogía. La edad de los estudiantes comprendía un rango entre 20 y 41 años $(M=$ 24.37 y $D T=3.38)$. La participación por parte de los estudiantes fue voluntaria y anónima. Respecto a la aplicación de los instrumentos, estos fueron aplicados en las clases tutoriales con la asistencia del profesor tutor.

\section{Instrumentos}

Los instrumentos utilizados en esta investigación son el test de Inteligencia creativa (CREA) de Corbalán Berná et al., (2003) para medir la creatividad. Para evaluar los estilos de pensamiento utilizamos el inventario de Estilos de Pensamiento revisado (TSI-R) (Sternberg et al., 2003) y para medir las estrategias de aprendizaje se utilizó la escala de Estrategias de Aprendizaje (ACRA) de Román y Gallego (2001).

El test $\boldsymbol{C R E A}$ (Corbalán Berná et al., 2003): es una medida cognitiva de la creatividad a través de la generación de preguntas a partir de un material gráfico suministrado. Este test está compuesto por tres tipos de pruebas o láminas: lámina A (a partir de 16 años), lámina B (12-16años) y lámina C (6-11 años). En la lámina A, que es utilizada en esta investigación, el sujeto ha de elaborar las preguntas de forma escrita. El test de inteligencia creatividad cumple con los criterios básicos de frabilidad y validez (Corbalan Berná et al., 2003).

E1 TSI-R (Sternberg et al., 2003): se conforma de 13 estilos intelectuales (legislativo, ejecutivo, judicial, monárquico, jerárquico, oligárquico, anárquico, global, local, interno, externo, liberal y conservador) agrupados en 5 dimensiones: i) funciones; ii) formas; iii) niveles; iv) ámbitos; v) tendencias. En este estudio se ha aplicado las subescalas de las funciones de los estilos de pensamiento: i) función legislativa; ii) función ejecutiva; iii) función judicial. Cada una de estas subescalas se conforman por 8 ítems de respuesta tipo Likert, donde la puntuación "7" se corresponde con "me describe totalmente" y " 1 " se corresponde con "no me describe en absoluto". Las diferentes escalas del TSI-R cumplen con los criterios de fiabilidad y validez (Sternberg et al., 2003; Zhang y Sternberg, 2005).

La escala ACRA (Román y Gallego, 2001): se sustenta en el cuerpo teórico de que los principales procesos cognitivos de procesamiento de la información. Estos son los procesos de adquisición, codificación-almacenamiento y recuperación, gobernados por otros de naturaleza metacognitivas denominados estrategias de apoyo. En este estudio se han utilizado las subescalas que corresponden a las estrategias metacognitivas lógicas: i) autoconocimiento de estrategias de adquisición y codificación y recuperación, con siete ítems; ii) automanejo de la planificación, con cinco ítems; iii) y auto- 
manejo de regulación y evaluación, con seis ítems. El formato de respuesta tipo escala Likert, con una gradación comprendida entre los valores " 1 " (el sujeto nunca realiza lo descrito por el ítem) y "4" (el sujeto siempre realiza lo descrito por el ítem). Esta escala cumple con los criterios básicos de fiabilidad y validez (Román y Gallego, 2001).

\section{Procedimiento de análisis}

Los datos se han analizado usando un procedimiento de modelización mediante ecuaciones estructurales con el programa AMOS 18. Este procedimiento permite analizar y probar un modelo teórico de relaciones directas e indirectas entre las variables de estudio (Cook y Campbell, 1979).

El procedimiento seguido es el siguiente:

i) Testeo de los modelos de medida para cada una de las escalas utilizadas (TSI-R y ACRA). Tal y como recomiendan Bentler (1990) y McDonald y Marsh (1990), la evaluación de bondad de ajuste debe realizarse en base a varios índices : a) Chi-cuadrado: valores asociados a $p$, no significativos indican un buen ajuste del modelo (Jöreskog y Sörbom, 1993); El índice comparativo de ajuste $(C F I)$ : valores superiores a .95 indican un buen ajuste del modelo (Hu y Bentler, 1998); Índice ajustado de bondad de ajuste (AGFI): valores superiores a .80 indican un buen ajuste (Catena, Ramos y Trujillo, 2003); error cuadrático medio de aproximación o raíz cuadrada de la media del error de aproximación (RMSE $A$ ): valores inferiores a .05 indican un buen ajuste del modelo (Browne y Cudeck, 1993).

ii) Estimación de la fiabilidad y la varianza extraída de cada constructo con el fin de evaluar si los indicadores especificados son suficientes en la representación de los mismos. La fiabilidad de constructo se considera adecuada cuando los valores son superiores de $.70 \mathrm{y}$ la varianza extraída cuando el valor es superior .50 (Hair, Anderson, Tatham y Black, 2008). Por otro lado, coeficientes de validez superiores .60 informan positivamente de la validez de los indicadores del modelo conceptual propuesto (Fornell y Larcker, 1981).

iii) Estimación del valor alfa de Cronbach para analizar la consistencia interna de las escalas. Respecto a la consistencia interna de la escala, valores superiores a .70 se consideran adecuados.

iv) Y finalmente, se evalúa el modelo estructural teórico de relaciones entre las variables presentadas. Los índices y valores de bondad de ajuste para el modelo estructural son los mismos que para los modelos de medida.

\section{Resultados}

Estudio de los modelos de medida (escalas TSI-R y ACRA)

Para validar la estructura de ambas escalas (estilos de pensamiento y estrategias de metacognitivas) se ha realizado un análisis confirmatorio mediante modelos de ecuaciones estructurales.

\section{Escala de estilos de pensamiento}

Respecto al modelo de medida de los estilos de pensamiento (ver Figura 2 y Tabla 1), los resultados de evaluación del modelo indican un buen ajuste global del modelo de medida en todos los índices (Tabla 2). Así, Chi-cuadrado presenta un valor asociado de $p$ no significativo $\left(\chi^{2}=258.326\right.$; D.F. $=249 ; p=.329 ; C M I N=1037)$. El valor del índice comparativo de ajuste $(C F I)$ presenta un valor .997. El valor de $A G F I$ es .889. Y el valor de RMSEA es de .014. Por lo tanto, estos resultados indican que los índices presentados indican un buen ajuste del modelo de medida a los datos empíricos.

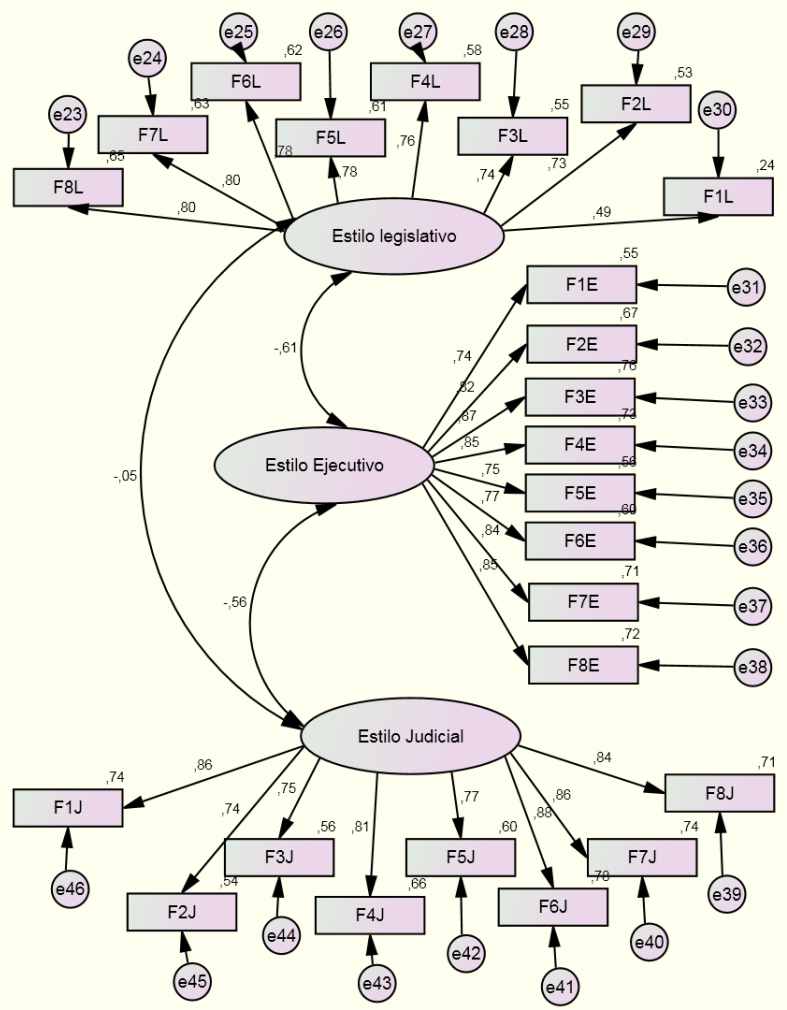

Figura 2. Modelo de medida de la escala de estilos de pensamiento.

Respecto a la fiabilidad de todos los constructos especificados (Tabla 3) supera el valor de .70, (S.L. = .91; S.E. $=$ .95; S.J. = .94). Asimismo, la varianza extraída (Tabla 3) es superior a $.50($ S.L. $=.55$; S.E. $=.63$; S.J. = .67). La mayoría de los coeficientes de validez de las saturaciones son superiores a .60, excepto "F1L" con un valor .49 (Figura 2). Por lo tanto, estos resultados indican que los indicadores empleados para explicar empíricamente los constructos latentes son adecuados. 
Tabla 1. Escala de Estilos de Pensamiento: Relaciones significativas y Pesos Estandarizados de Regresión.

\begin{tabular}{lcccccc}
\hline \multicolumn{2}{c}{ Relaciones entre } & S.E. & C.R. & $p$ & S.R.W. \\
\multicolumn{2}{l}{ Variables } \\
\hline F8L & $<---$ & E.L. & - & - & - & .803 \\
F7L & $<---$ & E.L. & .080 & 12.332 & $* * *$ & .796 \\
F6L & $<---$ & E.L. & .080 & 12.294 & $* * *$ & .785 \\
F5L & $<---$ & E.L. & .086 & 12.217 & $* * *$ & .784 \\
F4L & $<---$ & E.L. & .083 & 11.595 & $* * *$ & .759 \\
F3L & $<---$ & E.L. & .085 & 11.377 & $* * *$ & .744 \\
F2L & $<---$ & E.L. & .085 & 11.109 & $* * *$ & .727 \\
F1L & $<---$ & E.L. & .097 & 7.004 & $* * *$ & .493 \\
F1E & $<---$ & E.E. & - & - & - & .741 \\
F2E & $<---$ & E.E & .088 & 11.863 & $* * *$ & .821 \\
F3E & $<---$ & E.E. & .093 & 12.661 & $* * *$ & .869 \\
F4E & $<---$ & E.E. & .090 & 12.381 & $* * *$ & .852 \\
F5E & $<---$ & E.E. & .089 & 10.778 & $* * *$ & .750 \\
F6E & $<---$ & E.E. & .085 & 11.086 & $* * *$ & .772 \\
F7E & $<---$ & E.E. & .095 & 12.250 & $* * *$ & .843 \\
\hline & & & & & &
\end{tabular}

\begin{tabular}{llcllll}
\hline \multicolumn{3}{l}{ Relaciones entre } & S.E. & C.R. & $p$ & S.R.W. \\
\multicolumn{2}{l}{ Variables } \\
\hline F8E & $<---$ & E.E. & .091 & 12.260 & $* * *$ & .847 \\
F8J & $<---$ & E.J. & - & - & - & .845 \\
F7J & $<---$ & E.J. & .063 & 15.619 & $* * *$ & .862 \\
F6J & $<---$ & E.J. & .062 & 16.308 & $* * *$ & .882 \\
F5J & $<---$ & E.J. & .059 & 13.061 & $* * *$ & .773 \\
F4J & $<---$ & E.J. & .067 & 14.030 & $* * *$ & .810 \\
F3J & $<---$ & E.J. & .074 & 12.421 & $* * *$ & .747 \\
F2J & $<---$ & E.J. & .073 & 12.122 & $* * *$ & .735 \\
F1J & $<---$ & E.J. & .065 & 15.510 & $* * *$ & .859 \\
\hline
\end{tabular}

Nota: E.L., Estilo Legislativo; E.E., Estilo Ejecutivo; E.J., Estilo Judicial.; S.R.W., Pesos Estandarizados de Regresión; S.E., Error Estándar; C.R., Ratio Crítico.

Tabla 2. Índices de bondad de ajuste del modelo de medida

\begin{tabular}{clllll}
\hline$\chi^{2}$ & D.F. $p$ & CMIN/DF & CFI & AGFI & RMSEA \\
\hline
\end{tabular}

\begin{tabular}{llllllll}
\hline Modelo & 258.326 & 249 & .329 & 1037 & .997 & .889 & .014
\end{tabular}

Tabla 3. Modelo de medida de los estilos de pensamiento: Fiabilidad de constructos y varianza extraída.

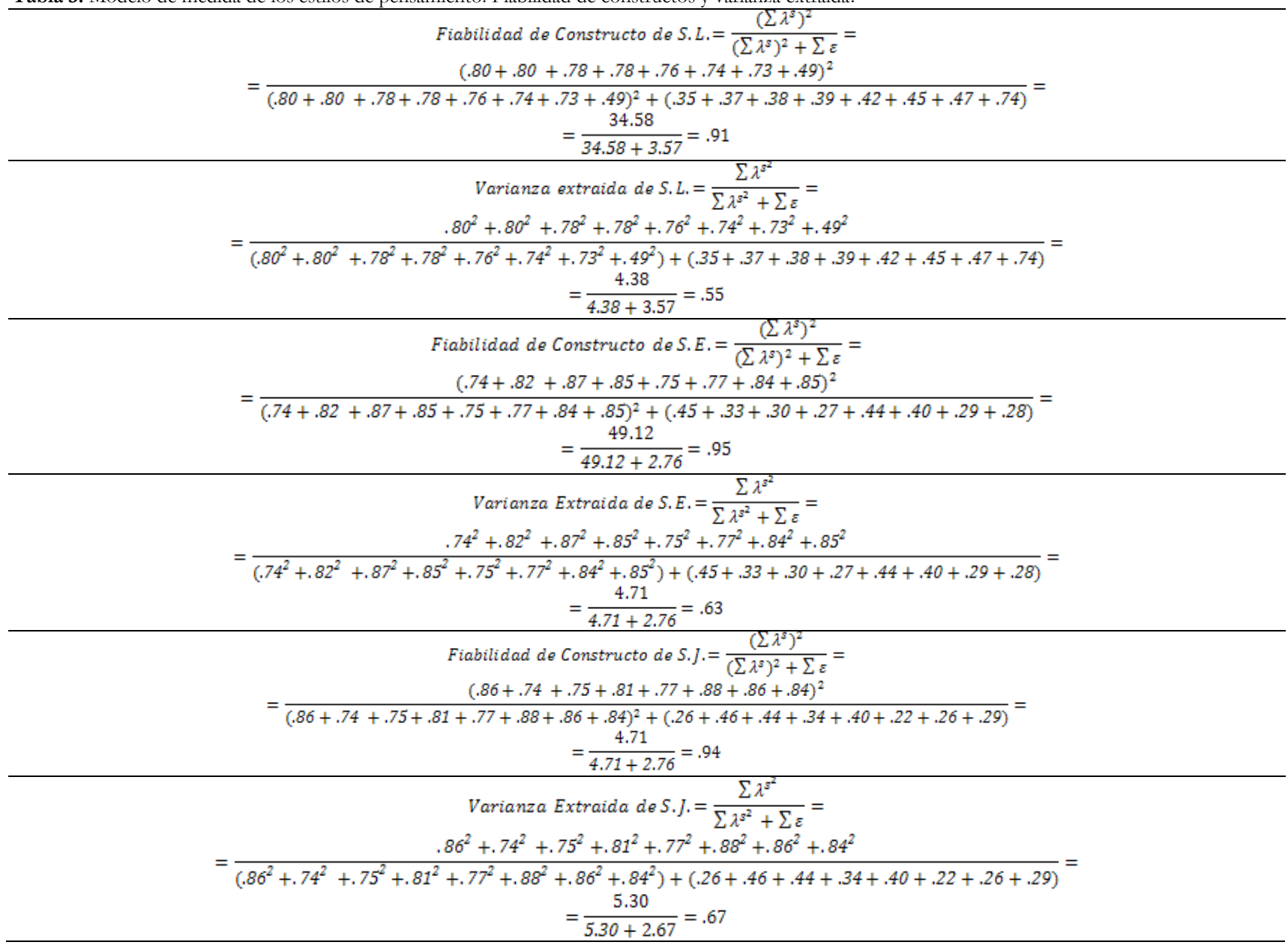

Para estudiar la fiabilidad de los instrumentos se calculó el coeficiente alfa de Cronbach (Tabla 4). La escala de estilos de pensamiento muestra valores adecuados para cada subescala. El estudio de incremento del alfa de Cronbach por eli- 
minación de ítems, indica que la eliminación de ninguno de los ítems mejora el valor de alfa (omitida por irrelevante).

Tabla 4. Confiabilidad de los factores que conforman la escala de estilos de pensamiento

\begin{tabular}{lll}
\hline Factores & alfa de Cronbach & Ítems-Total \\
\hline 1. Legislative Style & .927 & $n=8$ \\
2. Ejecutive Style & .941 & $n=8$ \\
3. Judicial Style & .940 & $n=8$
\end{tabular}

\section{Escala de estrategias metacognitivas}

Respecto al modelo de medida de las estrategias metacognitivas (ver Figura 3 y Tabla 5), los resultados de evaluación del modelo de medida indican un buen ajuste (Tabla 6). Así, Chi-cuadrado presenta un valor asociado de $p$ no significativo $\left(\chi^{2}=157.744 ;\right.$ gl. $\left.=132 ; p=.063 ; C M I N=1195\right) . \mathrm{El}$ valor del índice comparativo de ajuste $(C F I)$ respecto a un modelo nulo presenta un valor (.990). El valor de $A G F I$ es (.883). Y el valor de RMSE $A$ es de (.032). Por tanto, se observa un buen ajuste del modelo de medida a los datos empíricos.

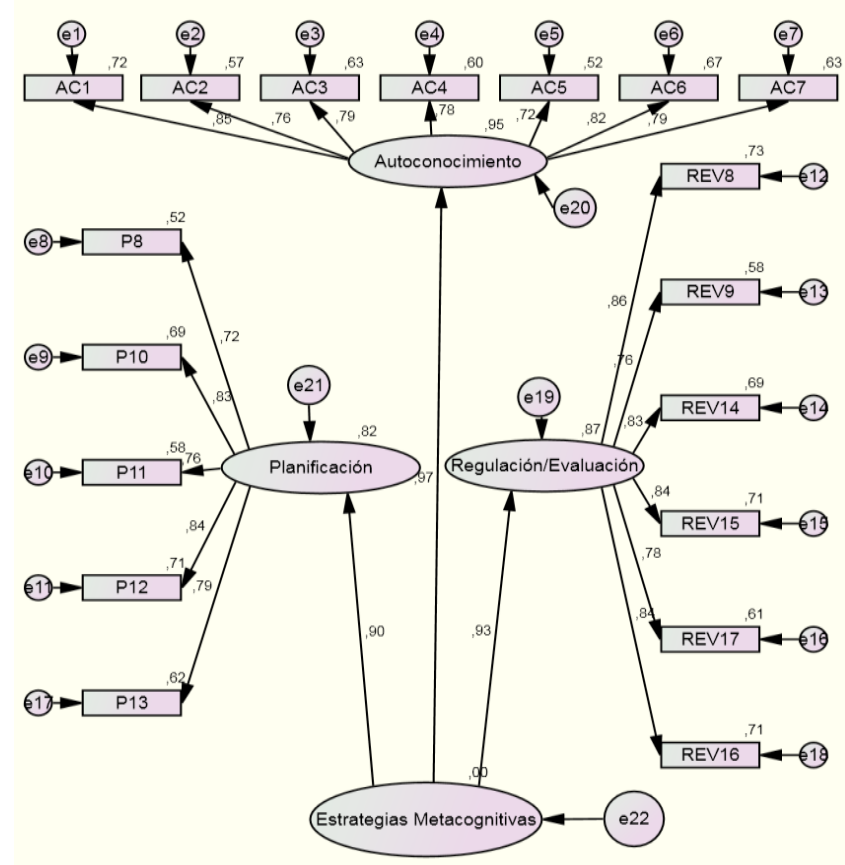

Figura 3. Modelo de medida de la escala de estilos de pensamiento.
Tabla 5. Escala de Estrategias Metacognitivas: Relaciones significativas y Pesos Estandarizados de Regresión.

\begin{tabular}{|c|c|c|c|c|c|c|}
\hline \multicolumn{3}{|c|}{$\begin{array}{l}\text { Relaciones entre } \\
\text { Variables }\end{array}$} & \multirow{2}{*}{$\begin{array}{c}\text { S.E. } \\
.073\end{array}$} & \multirow{2}{*}{$\begin{array}{l}\text { C.R. } \\
10.057\end{array}$} & & \multirow{2}{*}{$\begin{array}{r}\text { S.R.W } \\
.905\end{array}$} \\
\hline P. & $<---$ & E.M. & & & & \\
\hline A.C. & $<---$ & E.M. & .090 & 12.937 & *** & .973 \\
\hline R.E. & $<---$ & E.M. & - & - & - & .932 \\
\hline AC1 & $<---$ & A.C. & - & - & - & .848 \\
\hline AC2 & $<---$ & A.C. & .062 & 12.652 & $* * *$ & .756 \\
\hline AC3 & $<---$ & A.C. & .067 & 13.520 & $* * *$ & .792 \\
\hline AC4 & $<---$ & A.C. & .060 & 13.114 & $* * *$ & .775 \\
\hline AC5 & $<---$ & A.C. & .061 & 11.806 & $* * *$ & .722 \\
\hline AC6 & $<---$ & A.C. & .060 & 14.421 & $* * *$ & .821 \\
\hline AC7 & $<---$ & A.C. & .064 & 13.549 & $* * *$ & .794 \\
\hline P.8 & $<---$ & P. & - & - & - & .722 \\
\hline P.10 & $<---$ & P. & .108 & 11.176 & $* * *$ & .830 \\
\hline P.11 & $<---$ & P. & .110 & 10.346 & $* * *$ & .763 \\
\hline P.12 & $<---$ & P. & .107 & 11.494 & $* * *$ & .840 \\
\hline P.13 & $<---$ & P. & .111 & 10.637 & $* * *$ & .789 \\
\hline R.E.8 & $<---$ & R.E. & - & - & - & .857 \\
\hline R.E.9 & $<---$ & R.E. & .064 & 13.014 & $* * *$ & .764 \\
\hline R.E.14 & $<---$ & R.E. & .062 & 14.914 & $* * *$ & .832 \\
\hline R.E.15 & $<---$ & R.E. & .063 & 15.252 & $* * *$ & .841 \\
\hline R.E.17 & $<---$ & R.E. & .064 & 13.467 & $* * *$ & .781 \\
\hline R.E.16 & $<---$ & R.E. & .067 & 15.242 & $* * *$ & .840 \\
\hline
\end{tabular}

Nota: E.M., Estrategias Metacognitivas; P., Planificación; A.C., Autoconocimiento; R.E., Regulación y Evaluación; S.R.W., Pesos de Regresión Estandarizados; S.E., Error Estándar; C.R., Ratio Critico.

Tabla 6. Índices de bondad de ajuste: modelo de medida Estrategias Metacognitivas

\begin{tabular}{llllllll}
\hline & $\chi^{2}$ & gl. & $p$ & $C M I N / D F$ & $C F I$ & AGFI & RMSEA \\
\hline Modelo & 157.744 & 132 & .063 & 1195 & .99 & .883 & .032 \\
\hline
\end{tabular}

La fiabilidad de todos los constructos especificados (Tabla 7) supera el valor de .70 , (E.M. = .95; P.N. = .89; A.U. $=$ .91 ; R.E. $=.92)$. Asimismo, la varianza extraída (Tabla 7) es superior a .50 (E.M. $=.87$; P.N. = .62; A.U. $=.61$; R.E. $=$ .67). Los coeficientes de validez de las saturaciones son superiores a .60 (Figura 3). Así, estos resultados indican que los indicadores empleados para explicar empíricamente los constructos latentes son adecuados. 
Tabla 7. Estrategias Metacognitivas. Fiabilidad de Constructos y Varianza Extraída.

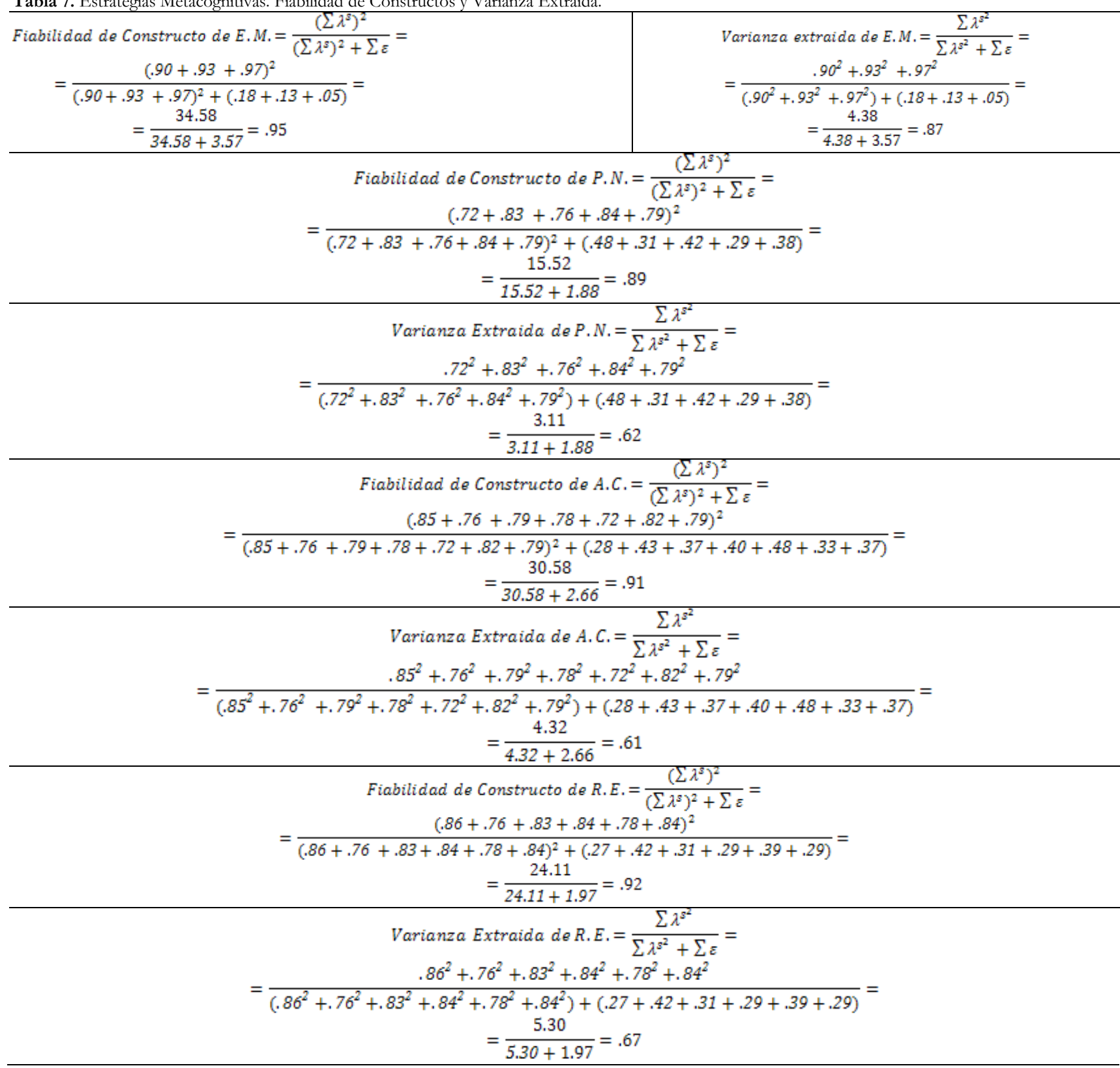

Respecto a la fiabilidad de las subescalas de estrategias metacognitivas, los resultados indican que todas las subescalas presentan valores superiores a .70 (Tabla 8). El estudio de incremento del alfa de Cronbach por eliminación de ítems, indica que no es posible mejorar el valor de alfa, con la eliminación de ítems (información omitida por irrelevante).

Tabla 8. Confiabilidad de los factores que conforman la escala de estrategias metacognitivas

\begin{tabular}{lll}
\hline Factores & alfa de Cronbach & Ítems-Total \\
\hline 1. Autonocimiento & .919 & $n=7$ \\
2. Planificación & .892 & $n=5$ \\
3. Regulación y evaluación & .924 & $n=6$ \\
\hline
\end{tabular}

En suma, el análisis confirmatorio indica que ambos modelos de medida (inventario de los estilos de pensamiento y escala de estrategias metacognitivas) son adecuados.

\section{Estudio del ajuste del modelo de estructura}

Los resultados de evaluación del modelo (Figura 4) muestran un buen ajuste global del modelo a los datos empíricos en todos los índices (Tabla 9). Así, Chi-cuadrado presenta un valor asociado de $p$ no significativo $\left(\chi^{2}=915.084\right.$; $D . F .=852 ; p=.066 ; C M I N=1074)$. El valor del índice comparativo de ajuste $(C F I)$ respecto a un modelo nulo presenta 
un valor de .99 . El valor de $A G F I$ es (.812). Y el valor de RMSE $A$ es de (.019). Por tanto, se observa un buen ajuste del modelo de medida a los datos empíricos.

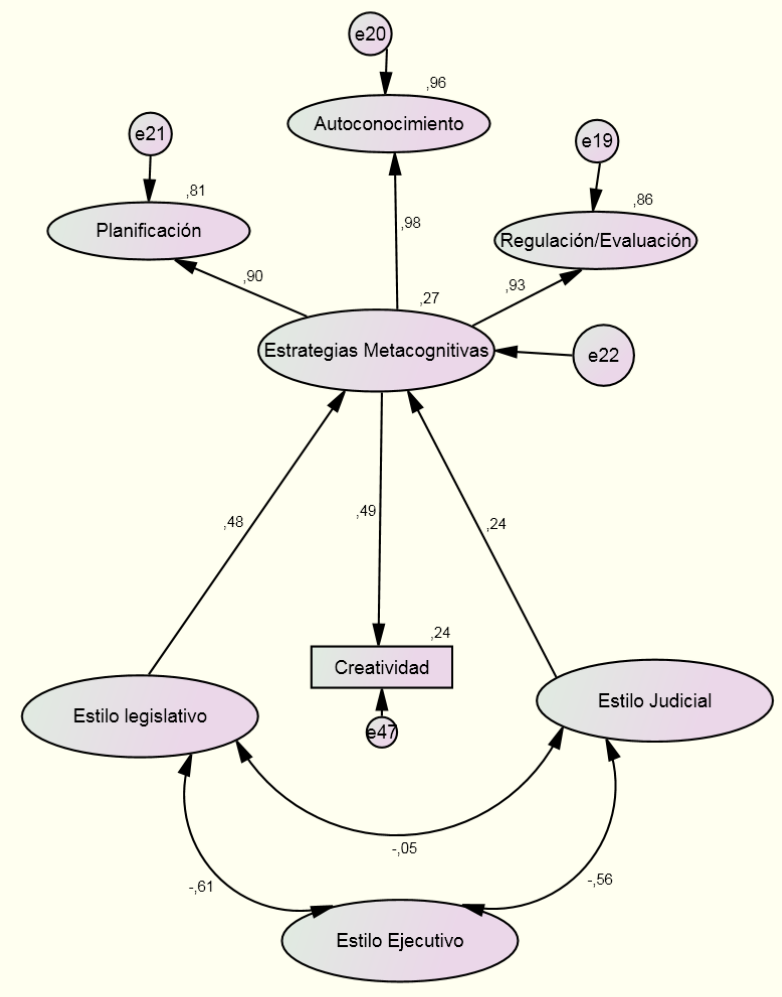

Figura 4. Relación entre estilos de pensamiento, estrategias metacognitivas y creatividad.

Nota: Se han ocultado los ítems de cada factor ya que fueron expuestos en apartados anteriores.

Tabla 9. Índices de bondad de ajuste: modelo de estructura.

\begin{tabular}{llllllll}
\hline & $\chi^{2}$ & gl. & $p$ & $C M I N / D F$ & $C F I$ & AGFI & RMSE $A$ \\
\hline Modelo & 915.084 & 852 & .066 & 1074 & .99 & .812 & .019
\end{tabular}

Como se observa (Tabla 10), las relaciones entre las variables apuntadas por el modelo teórico son significativas. Las estrategias metacognitivas se relacionan significativamente con dos estilos de pensamiento, el estilo de pensamiento judicial y, sobre todo, el estilo legislativo. Estas estrategias se relacionan significativamente con las estrategias de: autoconocimiento; planificación; y la regulación y evaluación. Del mismo modo, estas estrategias metacognitivas se relacionan con la creatividad. Sin embargo, no se han encontrado relaciones significativas entre los estilos de pensamiento y la creatividad.

Respecto a los efectos directos e indirectos (Tabla 10), se observa que: i) sobre las estrategias de autoconocimiento ( $\lambda$ $=.979)$, las estrategias de planificación $(\lambda=.902)$ y las estrategias de autorregulación $(\lambda=.927)$ contribuyen directa y positivamente las estrategias metacognitivas $(\lambda=.979 ; \lambda=.902$; $\lambda=.927$, respectivamente) e indirecta y positivamente los es-

tilos de pensamiento judicial $(\gamma=.236 ; \gamma=.218 ; \gamma=.224) \mathrm{y}$ legislativo $(\gamma=.466 ; \gamma=.429 ; \gamma=.441)$; ii) sobre las estrategias metacognitivas contribuyen directa y positivamente los estilos de pensamiento legislativo $(\gamma=.476)$ y judicial $(\gamma=$ .241); iii) sobre la creatividad contribuyen directa y positivamente las estrategias metacognitivas $(\lambda=.486)$ e indirecta $y$ positivamente los estilos de pensamiento legislativo $(\gamma=$ .231) y judicial $(\gamma=.117)$. Así, aunque no existe una relación significativa directa entre los estilos de pensamiento y la creatividad, se observa un efecto indirecto a través de las estrategias metacognitivas.

Tabla 10. Pesos de Regresión y pesos estandarizados de regresión.

\begin{tabular}{|c|c|c|c|c|c|}
\hline \multicolumn{2}{|c|}{$\begin{array}{l}\text { Relaciones entre } \mathrm{Va} \text { - } \\
\text { riables }\end{array}$} & \multirow{2}{*}{$\frac{\text { S.E. }}{.134}$} & \multirow{2}{*}{$\begin{array}{r}\text { C.R. } \\
3.196\end{array}$} & \multirow{2}{*}{$\frac{p}{* * *}$} & \multirow{2}{*}{$\frac{\text { P.E.R }}{.241}$} \\
\hline M.S. & $<---\quad$ S.J. & & & & \\
\hline M.S. & $<---\quad$ S.L. & - & - & - & .476 \\
\hline P.N.S. & $<---\quad$ M.S. & .040 & 5.806 & $* * *$ & .902 \\
\hline A.C.S. & $<---\quad$ M.S. & .060 & 6.227 & $* * *$ & .979 \\
\hline R.E.S. & $<--\quad$ M.S. & .051 & 6.231 & $* * *$ & .927 \\
\hline C.E. & $<---\quad$ M.S. & .423 & 4.951 & $* * *$ & .486 \\
\hline
\end{tabular}

Nota: M.S., Estrategias Metacognitivas; P.N.S., Planificación; A.C.S., Autonoconocimiento; R.E.S., Regulación y Evaluación; C.E., Creatividad; P.E.R., Pesos estandarizados de Regresión; S.E. Estimación del Error; C.R. Ratio Crítico.

Nota $* * *, p<.001$

Los efectos totales (Tabla 11) indican que el estilo de pensamiento que contribuye con más peso a las estrategias metacognitivas es el estilo legislativo $(\gamma=.476)$. Y la variable que influye con más peso sobre la creatividad son las estrategias metacognitivas $(\beta=.486)$.

Tabla 11. Efectos directos, indirectos y totales.

\begin{tabular}{llll}
\multicolumn{1}{l}{ Efectos } & Directo & Indirecto & Total \\
Sobre A.C. & & & \\
A.C.S. & - & - & - \\
P.N.S. & - & - & - \\
R.E.S. & - & - & - \\
M.S. & .979 & - & .979 \\
S.L. & - & .466 & .466 \\
S.E. & - & - & - \\
S.J. & - & .236 & .236 \\
Sobre P.N. & & & \\
A.C.S. & - & - & - \\
P.N.S. & - & - & - \\
R.E.S. & - & - & - \\
M.S. & .902 & - & .902 \\
S.L. & - & .429 & .429 \\
S.E. & - & - & - \\
S.J. & - & .218 & .218 \\
Sobre R.E. & & & \\
A.C.S. & - & - & - \\
P.N.S. & - & - & - \\
R.E.S. & - & - & - \\
M.S. & .927 & - & .927 \\
S.L. & - & .441 & .441 \\
S.E. & - & - & - \\
S.J. & - & .224 & .224 \\
& & &
\end{tabular}


Sobre S.M

A.C.S.

P.N.S.

R.E.S.

M.S.

S.L.

S.E.

S.J.

Sobre C.E.

A.C.S.

P.N.S.

R.E.S.

M.S.

S.L.

S.E.

S.J.

Nota: M.S., Estrategias metacognitivas; P.N.S., Planificación; A.C.S., Autonoconocimiento; R.E.S., Regulación y Evaluación; C.E., Creatividad; S.L., Estilo Legislativo; S.E., Estilo Ejecutivo; S.J., Estilo Judicial.

\section{Discusión}

Aunque existe un cuerpo empírico que explicita relaciones de dependencia y/o predictivas entre estos constructos, estas ofrecen explicaciones sobre relaciones únicas entre variables. Por ello, nos propusimos evaluar un modelo teórico que relaciona dichos constructos y explicita los efectos directos e indirectos mediante la modelización por ecuaciones estructurales.

Los hallazgos de nuestro trabajo indican que: i) las estrategias metacognitivas contribuyen directa y positivamente a la creatividad; ii) los estilos de pensamiento legislativo y judicial contribuyen directa y positivamente al uso de estrategias metacognitivas; iii) el efecto observado que ejercen estos estilos de pensamiento sobre la creatividad es indirecto y se produce por mediación de estrategias metacognitivas.

En base a estos resultados, discutimos el cuerpo empírico que ha fundamentado este estudio:

i) Relación entre los estilos de pensamiento y las estrategias metacognitivas: los resultados obtenidos, apoyan los hallazgos de Rosário et al., (2005); Zhang (2000) Y Zhang y Sternberg (2000) al indicar que exclusivamente los estilos judicial y legislativo se relacionan con las estrategias metacognitivas. Pero no apoyan el estudio de Zhang (2010a) que indica que: i) el estilo ejecutivo presenta una relación predictiva con dichas estrategias metacognitivas: autoconocimiento; planificación; y regulación y evaluación; ii) el estilo legislativo se relaciona exclusivamente con el componente autoconocimiento; iii) el judicial no presenta ninguna relación con dichas estrategias. Tampoco apoyan los resultados de Caycho (2009) en los que concluye que el uso de estrategias metacognitvias es independiente de la preferencia por uno u otro estilo de pensamiento.

ii) Relación entre los estilos de pensamiento y la creatividad: nuestros resultados apoyan las conclusiones de Almansa
Martínez y López Martínez (2010); López Martínez y Martin Brufau (2010); Zhang (2010b) y las afirmaciones de Sternberg (1999) y Zhang y Sternberg (2005). El matiz que aporta este estudio es que si bien existe un efecto entre dichos estilos y la creatividad éste es indirecto, y está mediado por las estrategias metacognitivas. Estos resultados no apoyan las conclusiones de Zhang (2010a) que indican que el estilo ejecutivo presenta una relación predictiva con la creatividad. Tampoco apoyan los resultados de Zhang (2004), que indican que el estilo ejecutivo se relaciona significativamente y de manera negativa con la creatividad. En nuestro estudio se observa nulidad de relación significativa, y ningún tipo efecto (directo o indirecto) entre dicho estilo y la creatividad.

iii) Relación entre estrategias metacognitivas y creatividad: los resultados indican un efecto directo de las estrategias metacognitivas sobre la creatividad. Estos apoyan, entre otros, los resultados de Chand y Runco (1992); Harrington (1975); Zhang (2010a); y las afirmaciones de Armbruster (1989); Pesut (1990); Mednick (1962); Rothenberg (1990); Runco (2007). Pero no, los resultados de Furnham et al., (2006) y Wolfradt y Pretz (2001), aunque tenemos que matizar que mientras estos dos estudios se realizaron con una muestra de estudiantes universitarios procedentes de la licenciatura de Bellas Artes, los participantes en nuestro estudio pertenecían a la licenciatura Psicopedagogía, y por lo tanto pertenecen a distintas disciplinas del conocimiento.

En suma, sin ánimo de generalizar debido a las características de la muestra de este estudio, con los resultados obtenidos se puede inferir que, sin la mediación de las estrategias metacognitivas, el comportamiento creativo puede surgir de forma casual, pero no de manera intencional y autocontrolada. Avanzamos, pues, en esas relaciones al encontrar hechos no observados hasta el momento.

Aún con las limitaciones expresadas, nos atrevemos a aceptar con cierta relatividad las conclusiones de Zhang (2010a) cuando afirma la existencia de una relación entre estilos ejecutivos y uso de estrategias metacognitivas y creatividad. Nosotros consideramos que la ausencia de innovación y aceptación del convencionalismo en la acción, características de las personas que actúan mediante estilos ejecutivos, no son comportamientos contrarios, sino ajenos al uso consciente y arriesgado de los modelos de pensamiento adquiridos, propios del dominio y uso de estrategias metacognitivas y el pensamiento creativo.

Para futuros estudios sería conveniente replicar esta investigación con muestras más amplias y diversificadas de estudiantes, procedentes de diferentes contextos universitarios y en distintos dominios específicos. Igualmente, serían relevantes estudios que profundizasen en las relaciones del modelo teórico probado con la creatividad conceptualizada desde enfoques personológicos. 


\section{Referencias}

Almansa Martínez, P. y López Martínez, O. (2010). ¿Existe relación entre creatividad y preferencia estilística en un grupo de alumnos de enfermería? Anales de Psicología, 26(1), 145-150.

Amabile, T. M. (1983). The social psychology of creativity. New York: Springer.

Armbruster, B. B. (1989). Metacognition in Creativity. En J. A. Glover, R. R. Ronning y C. R. Reynolds (Eds.), Handbook of creativity (pp. 177-182). New York: Plenum Press.

Barak, M. (2010). Motivating self-regulated learning in technology education. International Journal of Technology and Design Education, 20, 381-401.

Barron, F. (2007). Enhancement and the Fulfillment of Potential. En M. A. Runco, Creativity. theories and themes: research, development, and practice (pp. 319-374). San Diego, CA: Academic Press.

Bentler, P. M. (1990). Comparative fit indexes in structural models. Psychological Bulletin, 107, 238-246.

Bernal, T. (2001). Más allá de las cuentas: procesos cognitivos y estructuras auditivas. Proyecto Institucional, Facultad de Psicología. Universidad Santo Tomás, Bogotá

Biggs, J. B. (1987). Student Approaches to Learning and Studying. Hawthorn, Vic.: Australian Council for Educational Research.

Bransford, J. y Stein, B. (1984). The IDEAL problem solver: A guide for improving thinking, learning, and creativity. New York: W.H. Freeman.

Browne, M. W. y Cudeck, R. (1993). Alternative ways of assessing model fit. En K. A. Bollen y J. S. Long (Eds.), Testing Structural Equation Models (pp. 136-162). Beverly Hills, CA: Sage.

Catena, A., Ramos, M. M. y Trujillo, H. (2003). Análisis Multivariado: un manual para investigadores. Madrid: Biblioteca Nueva.

Caycho Rodríguez, T. P. P. (2009). Empleo de estrategias de aprendizaje según el estilo de pensamiento en adolescentes de ambientes empobrecidos. Revista de Psicología, 11, 66-78.

Chand, I. y Runco, M.A. (1992). Problem finding skills as components in the creative process. Personality and Individual Differences, 14, 155-162.

Clapham, M. M. y King, W.R. (2010). Psychometric Characteristics of the CREA in an English Speaking Population. Anales de Psicología, 26(2), 206-21.

Cook, T. D. y Campbell, D.T. (1979). Quasi-Experimentation: Design and Analysis for Field Settings. Chicago: Rand McNally.

Corbalán Berná, J., Martínez Zaragoza, F., Donolo, D., Alonso Monreal, C., Tejerina Arreal, M. y Limiñana Gras, M. R. (2003). CREA. Inteligencia Creativa. Una medida Cognitiva de la Creatividad. Madrid: TEA Ediciones.

Chrobak, R. y Prieto, A. P. (2010). La herramienta UVE del conocimiento para favorecer la creatividad de docentes y estudiantes. Anales de psicología, 26(2), 259-266.

Csikszentmihalyi, M. (1997). Finding Flow: The psychology of engagement with everyday life. New York: Basic Books.

Denckla, M.B. (2007). Executive function: Binding together the definitions of attention-deficit/hyperactivity disorder and learning disabilities. En L. Meltzer (Ed.), Executive function in education: From theory to practice (pp. 5-18). New York: The Guilford Press.

Einstein, A. (1985). Mi visión del mundo. Barcelona: Tusquets Editores.

Entwistle, N. y Ramsden, R. (1983). Understanding student learning. London: Croom Helm.

Feldhusen, J. F. y Goh, B. E. (1995). Assessing and accessing creativity: An integrative review of theory, research, and development. Creativity Research Journal, 8(3), 231-247.

Flavell, J. H. (1977): El desarrollo cognitivo. Madrid: Visor.

Fornell, C. y Larcker, D. (1981). Structural equation models with unobservable variables and measurement error. Journal of Marketing Research, 18(1), 39-50.

Furnham, A., Zhang, J. y Chamorro-Premuzic, T. (2006). Personality, art judgment, and creativity. Imagination. Cognition and Personality, 25, 119145.

Grigorenko, E. L. y Sternberg, R. J. (1997). Styles of thinking, abilities, and academic performance. Exceptional Children, 63, 295-312.

Guilford, J.P. (1950). Creativity. The American Psychologist, 5(9), 444-454.
Guilford, J.P. (1967). The nature of buman intelligence. New York: McGrawHill.

Hair, J.F., Anderson, R.E., Tatham, R.L. y Black, W.C. (2008). Análisis multivariante. Madrid: Prentice Hall.

Harrington, D.M. (1975). Effects of explicit instructions to "be creative" on the psychological meaning of divergent thinking test scores. Journal of Personality, 43(3), 434-454.

Hernández Pina, F. y Hervás Avilés, R.M. (2005). Enfoques y estilos de aprendizaje en educación superior. Revista española de orientación y psicopedagogía, 16(2), 283-299.

Hu, L.T. y Bentler, P.M. (1998). Fit indices in covariance structure modeling: sensitivity to underparameterized model misspecification. Psychological Methods, 3, 424-453.

Jausovec, N. (1994). Metacognition in creative problem-solving. En M. A. Runco (Ed.), Problem finding, problem solving, and creativity (pp. 77-95). Norwood, NJ: Ablex.

Jöreskog, K.G. y Sörbom, D. (1993). LISREL 8: Structural equation modeling with the SIMPLIS command language. Hillsdale, NJ: Lawrence Erlbaum Associates.

López Martínez, O. y Martín Brufau, R. (2010). Estilos de pensamiento y creatividad. Anales de psicología, 26(2), 254-258.

Martínez-Fernández, J. R., Villegas, M. E. y Martínez Torres, M. (2004). Concepciones de aprendizaje y estrategias metacognitivas en universitarios venezolanos y españoles. Revista Latina de Pensamiento y Lenguaje, 12(1), 21-35

McDonald, R.P. y Marsh, H.W. (1990). Choosing a multivariate model: noncentrality and goodness of fit. Psychological Bulletin, 107, 247-255.

Mednick, S.A. (1962). The associative basis of the creative process. Psychological Review, 69, 220-232.

Nickerson, R.S. (1999). Enhancing creativity. En R. J. Sternberg (Ed.), Handbook of creativity (pp. 392-430). New York: Cambridge University Press.

Oliveira, E., Almeida, L., Ferrándiz, C., Ferrando, M., Sainz, M. y Prieto, M.D. (2009). Tests de Pensamiento Creativo de Torrance (TTCT): Elementos para la validez del constructo en adolescentes portugueses. Psicothema, 21, 562-567.

O’Neil, H.F. y Abedi, J. (1996). Reliability and validity of a state metacognitive inventory: Potential for alternative assessment. The Journal of Educational Research, 89(4), 234-245.

Pesut, D.J. (1990). Creative thinking as a self-regulatory metacognitive process: A model for education, training and further research. Journal of Creative Behavior, 24, 105-110.

Román, J.M. y Gallego, S. (2001). ACRA: Escalas de Estrategias de Aprendizaje. Madrid: TEA Ediciones.

Rosário, P., Nuñez, J.C., González-Pienda, J.A., Almeida, L., Soares, S. y Rubio, M. (2005). El aprendizaje escolar examinado desde la perspectiva del "Modelo 3P" de J. Biggs. Psicothema, 17(1), 20-30.

Rothenberg, A. (1990). Creativity and madness: New findings and old stereotypes. Baltimore, M.D: Johns Hopkins University Press.

Rucinski, T.T. y Arredondo, D.E. (1994). Using the workshop approach in university classes to develop students dispositions to think metacognitively and creatively. Innovative Higher Education,18(4), 273288.

Runco, M.A. (1990). Implicit theories and creative ideation. En M. A. Runco y R. S. Albert (Eds.), Theories of creativity (pp. 234-252). Newbury Park, CA: Sage Publications.

Runco, M.A. (1993). Divergent thinking, creativity, and giftedness. Gifted Child Quarterly, 37, 16-22.

Runco, M.A. (2004). Creativity. Annual Review of Psychology, 55, 657-687.

Runco, M.A. (2006). Creativity is always personal and only sometimes social. En J. Schaler (Ed.), Howard Gardner under fire (pp. 137-150). Chicago: Open Court Publishers.

Runco, M.A. (2007). Creativity. Theories and themes: Research, development, and practice. San Diego, CA: Academic Press. 
Runco, M.A. y Okuda, S. M. (1988). Problem-discovery, divergent thinking, and the creative process. Journal of Youth and Adolescence, 17, 211-220.

Sternberg, R.J. (1985). Beyond IQ: A triarchic theory of buman intelligence. New York: Cambridge University Press.

Sternberg, R. J. (1988). Mental self-government: A theory of intellectual styles and their development. Human Development, 31, 197-224.

Sternberg, R. J. (1998). Estilos de pensamiento: claves para identificar nuestro modo de pensar y enriquecer nuestra capacidad de reflexión. Barcelona: Paidós.

Sternberg, R. J. (Ed.) (1999). Handbook of creativity. New York: Cambridge University Press.

Sternberg, R. J. y Lubart, T. I. (1996). Investing in creativity. American Psychologist, 51, 677-688.

Sternberg, R. J. y Lubart, T. I. (1997). La creatividad en una cultura conformista. Un desafio a las masas. Barcelona: Paidós.

Sternberg, R. J. Wagner, R. K. y Zhang, L. F. (2003). Thinking Styles Invento$r y$-Revised. Unpublished test, Yale University, New Haven, CT.

Torrance, E.P. (1966). Torrance Tests of Creative Thinking. Lexington, MA: Personnel Press.

Vermetten, Y., Vermunt, J., y Lodewijks, H. (1999). A longitudinal perspective on learning strategies in higher education: different viewpoints towards development. British Journal of Educational Psychology, 69, 221-242.

Wolfradt, U. y Pretz, J. (2001). Individual differences in Creativity: Personality, Story Writing and hobbies. European Journal of Personality, 15, 271310 .
Zampetakis, L.A. Bouranta, N. y Moustakis, V. (2010). On the relationship between individual creativity and time management. Thinking Skills and Creativity, 5(1), 23-32.

Zhang, L.F. (2000). Relationship between Thinking Styles Inventory and Study Process Questionnarie. Personality and Individual Difference, 29, 841856.

Zhang, L.F. (2004). Predicting cognitive development, intellectual styles, and personality traits from self-rated abilities. Learning and Individual Differences, 15, 67-88.

Zhang, L.F. (2010a). Do thinking styles contribute to metacognition beyond self-rated abilities? Journal of Psychology, 30(4), 481-494.

Zhang, L.F. (2010b). Do age and gender make a difference in the relationship between intellectual styles and abilities? European Journal of Psychology of Education, 25, 87-103.

Zhang, L.F y Sterberg, R. J. (2000). Are Learning Approaches and Thinking Styles related? A Study in Two Chinese Population. The Journal of Psychology, 134(5), 469-489.

Zhang, L.F. y Sternberg, R. J. (2005). A Threefold Model of Intellectual Styles. Educational Psychology Review, 17(1), 1-53.

(Artículo recibido: 01-04-2011, revisión: 20-09-2011, aceptado: 08-02-2012) 